\title{
Family Medicine and Disease Prevention
}

REVIEW ARTICLE

\section{Is Emotional Intelligence a Need Skill for Health Care Provider?}

\author{
Basem Abbas Al Ubaidi ${ }^{1,2 *}$ \\ ${ }^{1}$ Consultant Family Physician, Ministry of Health, Kingdom of Bahrain \\ ${ }^{2}$ Assistant Professor, Arabian Gulf University (AGU), Kingdom of Bahrain
}

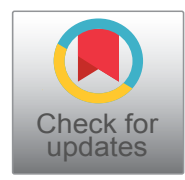

*Corresponding author: Basem Abbas Al Ubaidi, Consultant Family Physician, Ministry of Health; Assistant Professor, Arabian Gulf University (AGU), Kingdom of Bahrain, E-mail: bahmed1@health.gov.bh

\begin{abstract}
Health care providers are interested in understanding and applying the principles associated with emotional intelligence. There is decisive impact role in the practice of emotional intelligence skills and enhance social relationships, as well as improve health care and health care education. There are various ways that emotional intelligence skill can be taught, learned, and changed medical care programs for a better patient-doctor relationship.
\end{abstract}

\section{Keywords}

Emotional intelligence, Happiness, Mental health, Medical education, Professionalism, Graduate education

\section{Introduction}

Emotional intelligence interchangeably referred to as El, or Emotional Quotient (EQ) can apply to any social contact such as education, personal life, work and business [1]. It is one of the crucial factors that can predict life stability, psychological well-being, more over, in academic achievement $[2,3]$.

Higher EQ is related to the welfare of mental-social health performance [2].

Practicing medicine is involving many essential skills such as brilliant communicator, high-tolerance, wide-flexibility, decent-listener, smarter-worker and a passion for the practice $[4,5]$.

EQ is a critical building step for health care providers (HCWs) success, in particular, to manage the complexities inherent in the modern health care system is matching to invite career satisfaction [5].

HCPs need for both hard and soft skills, "hard skills" are the procedural skills, traditionally emphasised in formal training, while "soft skills" are the strategic skills such as interpersonal and communication skills and professionalism [5]. EQ construct is made up of the personal-emotional-social components of general intelligence [5]. Thus, EQ refers to a quantification of skills in practice [5].

Thus, creating a safe, non-judgmental, and conducive learning environment to practice EQ culture positively impacts on patient relationships, improve staff morale/recruitment/retention, healthier medical quality/safety/teamwork, enhance creativity, and commitment [5]. In contrary, poor application of EQ culture negatively harm the organisation's reputation, its patient care, and increase staff turn over [5].

Definition of emotional intelligence is, "an array of soft skills non-cognitive (emotional and social) capabilities, competencies, and skills that influence one's ability to succeed in coping with environmental demands and pressures" [6].

While EQ skills are not innate, it is the desire to learn self-improvement and willingness to have personal growth.

EQ has mostly learned skills- not innate genetic and need polish through experience and making proper changes, enhance through self-motivation, and improve over positive reinforcement feedback. EQ is essential and required in medical and non-medical professional working team and improve patient safety [57]. EQ is the ability to have successful, productive, and nurturing rapports; to strengthen carrier happiness; to reach personal goals; to turn plans into action; to make

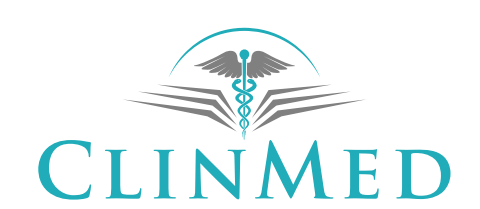

INTERNATIONAL LIBRARY
Citation: Al Ubaidi BA (2018) Is Emotional Intelligence a Need Skill for Health Care Provider?. J Fam Med Dis Prev 4:071. doi.org/10.23937/2469-5793/1510071

Received: April 03, 2017; Accepted: February 21, 2018; Published: February 23, 2018

Copyright: (C) 2018 Al Ubaidi BA. This is an open-access article distributed under the terms of the Creative Commons Attribution License, which permits unrestricted use, distribution, and reproduction in any medium, provided the original author and source are credited. 
informed decisions about the things that matter more than individual IQ [5-7].

EQ is the ability to recognise, appreciate, use, understand, and manage our emotions efficiently, to relieve interior strain, efficiently connect, empathise with others, overcome challenges, defuse conflict, understand people emotionally, and connect efficiently with them. EQ differs from $I Q$, in that $E Q$ can be learned through improving our social and emotional skills. Learning EQ not mean applying it, especially under tense stress and pressure; learning EQ expect, how to overcome and work under a stressful situation and remain emotionally stable. IQ can help in enhancing early academic years, but EQ is most important in personal, social relationship, even guarantee work and academic success. IQ and EQ exist in the cycle and each build and efficiently elevate others [5-8].

\section{Discussion}

EQ has two fundamental attributes which were positively or negatively affected each other: Self-improvement in our work which is personal competence and the second one is social competence (Figure 1) [5].

Personal competence divided into categories of self-perception; self-expression/regulation; self-management; and self-motivation for decision making. Moreover, social competenceis composed of social awareness; social empathy; interpersonal skills; social responsibility; and relationship management $[4,5]$.

Self-perception relies on having a deep understanding of our self-regard, emotional self-awareness (e.g. feeling, reaction, strengths, weakness, needs), and self-actualisation (e.g. how its effect on thoughts, behaviour and performance) $[5,6]$.

a) Self-regard is the ability to maintain self-respect with ethically stranded principles and values to sustain honesty of self, personal identity, and a feeling of person- confidence.

b) Emotional self-awareness is the ability to a norm and values of feelings and their impacts on the thoughts and takes actions to support oneself and others through mindfulness without judgment in difficult times (e.g. understand how/why one is feeling and teach how those feelings are similar to and dissimilar from the opinions of others).

c) Self-actualization is "the ability to understand the triangle of thought- behaviour- performance to maintain a precious, meaningful, purposeful and enjoyable life, by a willingness to improve oneself maximum aptitudes.

Self-expression which relies on having a deep understanding of oneself and others emotional expression, assertiveness, and independence [5-7].

a) Emotional expressionis a fruitful expression of emotions based on an ability to openly, convey one's feelings both verbally and non-verbally in a constructive way.

b) Assertiveness is the ability to transfer moods, opinions, and thoughts openly in a socially acceptable, non-destructive, and non-offensive manner.

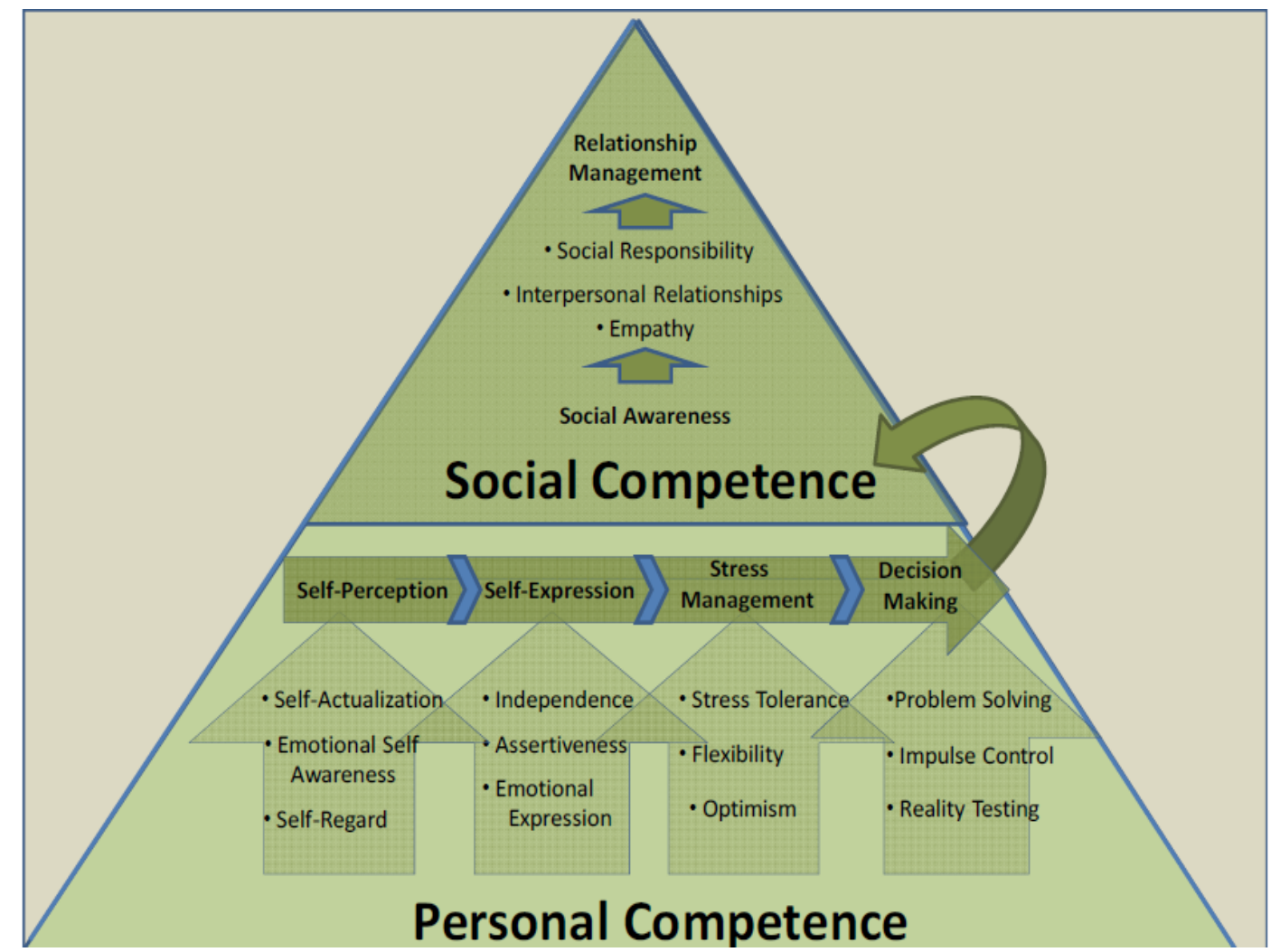

Figure 1: The Model of EQ HCWs characteristics. 
c) Independence is the ability to autonomously engage in decision making, planning, and self-regulation on daily tasks by being self-controlled and self-directed in one's actions.

Self-stress management characterised by the ability to handle any stressful situations without becoming overwhelmed (e.g. control impulsive behaviour, manage emotions in constructive ways) contains three sub-components: Optimism, stress tolerance, and flexibility [5-7].

a) Optimism is a positive attitude, remaining resilient and hopeful despite occasional setbacks by seeing the light at the end of the tunnel.

b) Stress tolerance is the resilience ability to withstand adverse events and stressful situations without falling apart by actively and positively coping with stress.

c) Flexibility is the ability to adjust one's thoughts, emotions, and behaviour to compelling circumstances that are unpredictable or unfamiliar.

Decision making is the fourth vital area of Personal Competence, containing three sub-elements: Reality testing, impulse control, and problem-solving [5-7].

a) Reality testingis the person's capacity to remain objectively size up by seeing things as they are, and considering the perspectives, needs, biases and beliefs that motivate them.

b) Impulse control is the skill to resist or delay impulse-angry outbursts, emotional tempers, passive-aggressive, irresponsible behaviour, insensitive statements, vindictiveness, incivility or uncontrolled passions.

c) Problem-solvingis the ability to generating, implementing and find practical solutions to problems in situations where emotions are involved, problem-solving has seven steps, including [5-7]

$\checkmark$ Identifying problem existence

$\checkmark$ Gathering the necessary information (both subjectively and objectively)

$\checkmark$ Generate a list of solutions (assessment/analysis)

$\checkmark$ Evaluate alternatives

$\checkmark$ Choosing an optimal solution

$\checkmark$ Implementing planning

$\checkmark$ Assessing the outcome

Social competence is a complex of a combination of social awareness and relationship management that allows the leader to understand the emotional theme of HCWs group, to communicate efficiently and compassionately with members of the team, and to solicit input from them [5-7].

Social awareness is the ability to understand social networks emotions, feelings, needs, and concerns of the
HCWs, appreciating group's values and culture through picking up verbal and nonverbal cues and enhance the power of group dynamics [5-7].

a) Empathy is the ability to recognise, understand HCWs and appreciate their feelings.

b) Interpersonal relationships based on developing and maintaining mutually satisfying relationships that are characterised by trust and compassion.

c) Social responsibility is the ability to have the sense of social consciousness and to show concern and sharing others social interests, obligations and reflects behaviours of willingly contributing to society and generally to the welfare of others.

Relationship managementis to develop, maintain to and nurtures interactions with HCWs to create a healthy environment, while constructive behaviour can be directed towards a definite efficiently to solve difficult challenges and to build bonds widely, inspire and influence HCWs, and to foster information sharing and creativity in HCWs [5-7].

Happiness is the ability to feel satisfied, enjoy life and to have sufficient fun, happiness has a positive impact on work culture and improves work performance [5-7].

Highly achiever HCWs are characterised by having high motivation, intense drive and passion for working hard, which has an attractive team, infectious character, and powerful leadership charisma [5-7].

\section{EQ Paybacks to Physician, Patient, Organisation}

EQ is simple, practical skills for promoting efficiently and effectively connect with patients. The effective empathic expression does not take more time $[9,10]$, but when empathetic skills omitted, patients will repeat more needs and need more time [10]. EQ should be learned during medical training to have more connection with the patient to have a healer role for the physician [10]. True empathy is not about action; it is about a feeling, it is fully human, to care; it reduces patient anxiety, improves clinical outcomes and reduces malpractice claims [11,12].

Empathy is being aware of the feeling of others by reading and managing other's emotion. Therefore, empathy considered as an essential aspect of EQ [13]. Female had higher empathy than male scores and overall EQ [14-16].

Female medical students had higher EQ than males while in clinician on only one subscale of El, namely, stress management [14-17]. The more expert physician had more EQ than the junior physician [18-20].

EQ shows provider better patient-centred care [20], by increase patients' trust, which consequently increases patient satisfaction [17-19]. There is a positive correlation between EQ in conjunction with communication, interpersonal skills and teamwork effectiveness $[13-16,21,22]$. 
EQ may improve academic performance and clinical diagnostic, prognostic abilities, as well as a doctor-patient relationship $[23,24]$. There is a role between 'emotionally intelligent organisation [20] and decrease the impact of stress and burn out [25], workplace stress and depression. The emotionally intelligent organisation has increased organisational commitment, and raise organisation leadership [26].

There are direct links between EQ and six important medical competences (Interpersonal and Communication Competence, Practice-based Competence, improved Medical Knowledge Competence, Patient Care Competence, Systems-based Practice and Professionalism) [27].

Apply the concept of EQ, enhance patient-centred care and applying skills of EQ among health care providers, have more successful performance, more important outcomes in the workplace and improved individual behaviours. The success of implementing EQ skills requires staff commitment, the culture of communication, collaboration and adaptability.

Physician empathy improves patient satisfaction, adherence to treatment, fewer medical errors, and better at managing chronic conditions [28].

EQ publicised to absolutely contribute to the physician-patient relationship, increased empathy, teamwork, communication, stress management, organisational commitment, physician and nurse career satisfaction, and effective leadership.

There is a connection between EQ and patient care and physician career success, to develop an innovative behavioural construct to patient centred care [28].

$E Q$ helps physicians in changing self-devastating disruptive behaviour, indulge self-stress relief. It helps understand self-feelings, and follow optimal dreams. EQ is very powerful detector to recognise, direct and positively express emotions, build strong relationships and change behaviour. "Being a good physician is not only you equipped with technical knowledge but also require an understanding client's emotion [5-7]".

The ability to remain emotionally conscious and to keep nervous system in its comfort zone also ensure enhancing the immune system, and preserve and repair the damage [5-7].

If HCWs feel secure, calm, focused, creative, compassionate, and socially engaging, lead to the efficient work environment, in contrary feeling threatened, the body response will be in a resistive state of 3Fs (Fight, Flee, or Freeze) [5-7].

\section{EQ as a Vital Component to Medical Education}

Students with intrapersonal or interpersonal problems can suffer both academically and non-academically unable to succeed with the innovative, interactive learning and participation due to personal internal conflicts, anxiety, difficulties interacting with professors, colleagues, clinicians, and ultimately patients [29].

Students must see El as an integral part of their existing program and not as something to be developed in a separate environment [30,31].

Practical understanding and managing emotions ability to think abstractly non-cognitive qualifications and competencies cope with environmental demands and stressors [30,31].

The ability to perceive emotions is to access and produce feelings. To support thought, to grow emotionally and to adjust feelings. To promote expressive mood and intellectual growth [31]. To explain the psychological differences in individuals' abilities which is related to emotional engage in complex process concerning about their own and others feeling, as well as to use that information to guide thoughts and behaviour [30,31].

EQ contributes to an individual's ability to adjust socially, successfully work in a team, perform better, and cope more effectively with stress and environmental pressure [32-34]. Medical students with high EQ have less conflict in their interactions with both colleagues and superiors [35].

In the past, traditional medicine has encouraged HCPs to preserve an emotional distance from their patients, [36] while now a days; there have been breach barriers of communication between patients and HCPs, in favour of a more empathic approach. The relationship between patients and HCPs grow into more partnership, HCPs need to adopt good communication skills to improve patient satisfaction and to build mutual understanding [30].

HCPs must be competent in EQ and able to recognise shifts in a patient's moods and demean our early [30]. If HCPs understands the patient's background and emotional reactions, the medical advice and treatment can be custom-made to match the individual's expectations [30].

\section{Implication}

EQ test should be applied in premedical college selection criteria to have the best candidate [21]. Training medical students in EQ [37]. EQ related skills are of paramount importance of non-technical skills (e.g. perceiving, understanding, using, and managing emotions) can be improved through medical training. EQ programs must have established ways to implement, monitor, and evaluate the practice [30]. To have an EQ assessment and evaluation based on higher conceptual, theoretical and methodological framework for the clinical and academic performance [21].

\section{Conclusion}

There are general agreements that EQ skills are need- 
ed rather than pleasant to have it. EQ improves patient care outcome; through patient-centred care; improve the doctor-patient relationship. EQ enhances physician and patient well-being, expand patient safety, and gain healthier HCPs teamwork. IQ is neither the only issue in the making of a successful HCPs nor thesingle predictor of works success.

\section{Potential Conflicts of Interest}

None.

\section{Competing Interest}

None.

\section{Sponsorship}

None.

\section{References}

1. Amundson SJ (2005) The impact of relational norms on the effectiveness of health and human service teams. Health Care Manag 24: 216-224.

2. Arora $\mathrm{S}$, Ashrafian $\mathrm{H}$, Davis $\mathrm{R}$, Athanasiou $\mathrm{T}$, Darzi $\mathrm{A}$, et al. (2010) Emotional intelligence in medicine: a systematic review through the context of the ACGME competencies. Med Educ 44: 749-764.

3. Arora S, Sevdalis N, Nestel D, Tierney T, Woloshynowych $M$, et al. (2009) Managing intraoperative stress: what do surgeons want from a crisis training programme? Am J Surg 197: 537-543.

4. Austin EJ, Evans P, Goldwater P, Potter V (2005) A preliminary study of emotional intelligence, empathy and exam performance in first-year medical students. Pers Individ Dif 39: 1395-1405.

5. Austin EJ, Evans P, Magnus B, O'Hanlon K (2007) A preliminary study of empathy, emotional intelligence and examination performance in MBChB students. Med Educ 41: 684-689.

6. Bar-On R (2001) Emotional intelligence and self-actualisation. In: Ciarrochi J, Forgas JP, Mayer J, Emotional Intelligence in Everyday Life. A Scientific Inquiry. Psychology Press, 82-97.

7. Birks YF, Watt IS (2007) Emotional intelligence and patient-centred care. J R Soc Med 100: 368-374.

8. Bleakley A (2006) Broadening conceptions of learning in medical education: the message from teamworking. Med Educ 40: 150-157.

9. Brannick MT, Wahi MM, Arce M, Johnson HA, Nazian S, et al. (2009) Comparison of trait and ability measures of emotional intelligence in medical students. Med Educ 43: 1062-1068.

10. Del Canale S, Louis DZ, Maio V, Wang X, Rossi G, et al. (2012) The Relationship Between Physician Empathy and Disease Complications. Acad Med 87: 1243-1249.

11. Fernandez CSP, Peterson HB, Holmstrőm SW, Connolly AM (2012) Developing Emotional Intelligence for Healthcare Leaders, Emotional Intelligence - New Perspectives and Applications.

12. Johnson DR (2015) Emotional intelligence as a crucial component to medical education. Int J Med Educ 6: 179-183.

13. DiMatteo MR, Hays RD, Prince LM (1986) Relationship of physicians' nonverbal communication skill to patient satisfaction, appointment noncompliance, and physician workload. Health Psychol 5: 581-594.

14. Goleman G (1996) Emotional Intelligence: Why It Can Matter More Than IQ. Bloomsbury Publishing.

15. Hendren RL (1988) Predicting success and failure of medical students at risk for dismissal. J Med Educ 63: 596-602.

16. Humphreys J, Brunsen B, Davis D (2005) Emotional structure and commitment: implications for healthcare management. J Health Organ Manag 19: 120-129.

17. Kroenke K (2009) Unburdening the difficult clinical encounter. Arch Intern Med 169: 333-334.

18. Lopes PN, Brackett MA, Nezlek JB, Schütz A, Sellin I, et al. (2004) Emotional intelligence and social interaction. Pers Soc Psychol Bull 30: 1018-1034.

19. Lopes PN, Grewal D, Kadis J, Gall M, Salovey P (2006) Evidence that emotion-al intelligence is related to job performance and affect and attitudes at work. Psicothema 18: 132-138.

20. Lopes PN, Salovey P, Coté S, Beers M (2005) Emotion regulation abilities and the quality of social interaction. Emotion 5: 113-118.

21. Mansouri B (2001) Normalization of Sibria Shrink emotional Intelligence Test for students of MA public universities based in Tehran. MS thesis, evaluation and assessment. Allameh Tabatabaei University.

22. McCallin A, Bamford A (2007) Interdisciplinary teamwork: is the influence of emotional intelligence fully appreciated? J Nurs Manag 15: 386-391.

23. McQueen AC (2004) Emotional intelligence in nursing work. J Adv Nurs 47: 101-108.

24. Mikolajczak M, Menil C, Luminet O (2007) Explaining the protective effect of trait emotional intelligence regarding occupational stress: Exploration of emotional labour processes. Journal of Research in Personality 41: 1107-1117.

25. Pagnini F, Manzoni GM, Castelnuovo G (2009) Emotional intelligence training and evaluation in physicians. JAMA 301: 600 .

26. Pellitteri J (2002) The relationship between emotional intelligence and ego defense mechanisms. J Psychol 136: 182-194.

27. Petrides KV, Frederickson N, Furnham A (2004) The role of trait emotional intelligence in academic performance and deviant behaviour at school. Pers Individ Dif 36: 277-293.

28. Levinson W, Roter DL, Mullooly JP, Dull VT, Frankel RM (1997) Physician-Patient Communication. The Relationship with Malpractice Claims Among Primary Care Physicians and Surgeons. JAMA 277: 553-559.

29. Roter DL, Hall JA, Kern DE, Barker LR, Cole KA, et al. (1995) Improving physicians' interviewing skills and reducing emotional distress: a randomized clinical trial. Arch Intern Med 155: 1877-1884.

30. Salovey P, Mayer JD (1989) Emotional Intelligence. Imagination Cognition and Personality 9: 185-211.

31. Stein SJ, Book HE (2006) The EQ edge: emotional intelligence and your success. ( $2^{\text {nd }}$ edn), Toronto, Canada: MultiHealth Systems.

32. Stein SJ (2011) The Complete EQ-I 2.0 Model (technical manual). Toronto, Canada: Multi-Health Systems. 
33. Stratton TD, Elam CL, Murphy-Spencer AE, Quinlivan SL (2005) Emotional intelligence and clinical skills: preliminary results from a comprehensive clinical performance examination. Acad Med 80: S34-S37.

34. Stratton TD, Saunders JA, Elam CL (2008) Changes in medical students' emotional intelligence: an exploratory study. Teach Learn Med 20: 279-284.

35. Wagner PJ, Moseley GC, Grant MM, Gore JR, Owens C
(2002) Physicians emotional intelligence and patient satisfaction. Fam Med 34: 750-754.

36. Weng HC, Chen HC, Chen HJ, Lu K, Hung SY (2008) Doctors emotional intelligence and the patient-doctor relationship. Med Educ 42: 703-711.

37. Weng HC (2008) Does the physician's emotional intelligence matter? Impacts of the physician's emotional intelligence on trust, patient-physician relationship, and satisfaction. Health Care Manage Rev 33: 280-288. 